ORIGINAL ARTICLE

Luis Alexandre Piteira Gomes •

Pedro Miguel Pedreirinho Salgado •

Eduardo Nuno Barata • António Paulo Pereira Mira

\title{
Alarm scent-marking during predatory attempts in the Cabrera vole (Microtus cabrerae Thomas, 1906)
}

Received: 25 July 2012/ Accepted: 26 December 2012/Published online: 18 January 2013

(C) The Ecological Society of Japan 2013

\begin{abstract}
The alarm pheromones often released by animals under stressful situations seem to elicit behavioral changes in conspecifics, which in the appropriate context can be viewed as anti-predatory responses. However, the releasing of alarm pheromones associated with predatory events has not been demonstrated in mammals. In the current study with wild-caught Cabrera voles, we carried out experiments in the laboratory and in the field to assess the release of alarm pheromones in scent-marks during simulated predatory events and disclose their effects on conspecifics. We first conducted an assay wherein voles where let to scent-mark a clean substrate in the absence of disturbance (control) and under the simulation of predatory events. Contrarily to the control, no fecal boli were released and the area marked with urine was significantly larger during the predatory simulation. In a subsequent assay, we assessed the voles' preference between urine-marks released under predatory simulation and in control conditions. Voles showed a significant preference by control substrates. Finally, a third assay was carried out in the vole's habitat wherein the individual activity was monitored by radio-tracking before and after placement of urine-marks obtained during the conditions described above. The vole's activity was only reduced near the urine-marks released
\end{abstract}

\section{A. P. Gomes ( $₫)$}

Grupo de Ecossistemas e Paisagens Mediterrânicas, Instituto de Ciências Agrárias e Ambientais Mediterrânicas, Universidade de Évora, Núcleo da Mitra, Apartado 94, 7002-554 Évora, Portugal E-mail: luispgomes $@$ gmail.com

Tel.: +351-91-9957102

L. A. P. Gomes · P. M. P. Salgado · A. P. P. Mira Unidade de Biologia da Conservação, Universidade de Évora, Núcleo da Mitra, Apartado 94, 7002-554 Évora, Portugal

E. N. Barata

Departamento de Biologia, Universidade de Évora, Apartado 94, 7002-554 Evora, Portugal

A. P. P. Mira

CIBIO, Centro de Investigação em Biodiversidade e Recursos Genéticos, Pólo de, Evora, Portugal during the simulated predatory events. The results suggest that: (1) during predatory attempts, Cabrera voles release an alarm pheromone in their urine-marks; (2) the putative alarm pheromone reduces the voles' activity in the surroundings of the marked area; (3) the putative alarm pheromone persists in the field affecting conspecifics' activity for several days.

Keywords Alarm pheromone $\cdot$ Voles $\cdot$ Microtus cabrerae Scent-marking $\cdot$ Predation $\cdot$ Anti-predatory

\section{Introduction}

Many animals emit alarm signals in response to a stressful situation, particularly on the approach of predators or under a predatory attempt (Wyatt 2003; Caro 2005; Hollén and Radford 2009). These alarm signals may be directed to conspecifics in order to warn them of an impending danger and promote anti-predatory responses and/or directed to predators in order to deter them or attract additional ones, thus disrupting the predation event (Sherman 1985; Mathis and Smith 1992; Mathis et al. 1995; Wyatt 2003; Caro 2005). Often warning alarm signals' evolution had been viewed as one of the most puzzling questions in behavioral ecology since alarm signaling has a relative high cost to the signaler but no obvious direct benefits (Williams 1964, 1992; Taylor et al. 1990; Mathis et al. 1995). Nevertheless, some of these signals may have delayed benefits for signalers, such as saving the lives of individuals who will reciprocate in the future (Trivers 1971; Ashton et al. 1998), of potential mates (Witkin and Fitkin 1979), or other group members in circumstances where group living is beneficial (Smith 1986). Moreover, truly altruistic signals may have evolved in situations where the benefits fall on related individuals (Sherman 1977; Mathis et al. 1995; Wu et al. 2010).

The alarm signals may take many forms, being mostly visual, auditory, or chemical. Some of the prey use chemical alarm signals to convey information to 